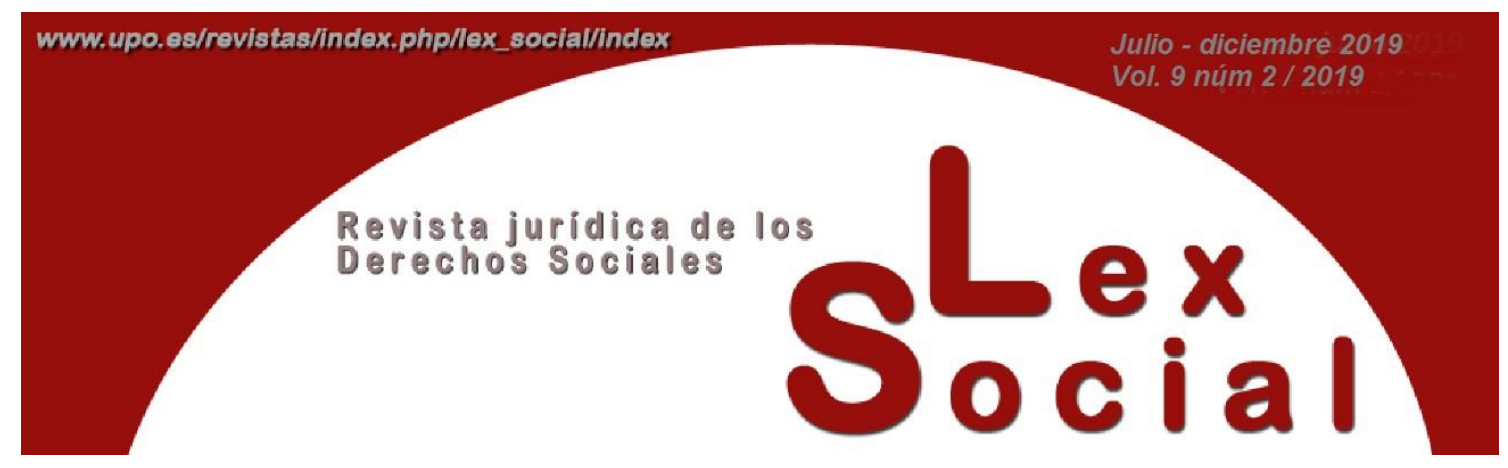

\title{
LA IMPORTANCIA DE LOS PISOS DE PROTECCIÓN SOCIAL PARA LOS ADULTOS MAYORES EN MÉXICO
}

\section{THE IMPORTANCE OF SOCIAL PROTECTION FLOORS FOR OLDER ADULTS}

\author{
GABRIELA MENDIZÁBAL BERMÚDEZْ \\ Profesora Titular $C$ Derecho \\ Universidad Autónoma del Estado de Morelos, México
}

Artículo recibido el 13 de mayo de 2019

Artículo aceptado el 17 de mayo de 2019

\section{RESUMEN}

El complejo mundo laboral, cada vez más, excluye a los adultos mayores. En América Latina la oferta laboral para este grupo etario tiende a ser menor, ya que los perciben como incapacitados para desempeñar algunas actividades, motivo por el cual les cierran las puertas limitando entre otros su derecho a la seguridad social. Por tales consideraciones resulta importante que se tomen medidas direccionadas a la solución de dichos problemas.

El presente artículo muestra un análisis del impacto de la iniciativa internacional del piso de protección social en beneficio de las personas adultas mayores, analizando aspectos referentes al aseguramiento social y la situación pensionaria a la que se enfrentan los adultos mayores. Por otro lado, se analiza la importancia y el papel que toman los pisos de protección social con referencia a los adultos mayores en México y los principales programas asistenciales que les otorgan protección social.

- Miembro del Sistema Nacional de investigadores nivel II. 
Palabras Clave: pisos de protección social, adultos mayores, seguridad social, protección social, pensiones, salud.

\begin{abstract}
The complex world of work increasingly excludes older adults. In Latin America, the labor supply for this group tends to be lower since they perceive them as incapacitated to perform some activities; because of this, they close their doors, denying them, among other rights, their right to social security. Due to this situation, it is important to take action to solve these problems.

This article shows on one side, the analysis of the impact of international initiative of the social protection floor for the benefits of older adults, evaluating aspects related to social insurance, such as pensions and health for the elderly. On the other side, it analyses the importance and role of social protection floors with reference to older adults in Mexico and the main welfare programs that provide social protection.
\end{abstract}

KEYWORDS: social protection floors, older adults, social security, social protection, pensions, health.

SUMARIO

\title{
I. Introducción
}

II. Marco teórico

II.1. Adultos mayores

II.1.1. Marco socio-demográfico de los adultos mayores en México

II.2. El piso de protección social

III. Seguridad social vs. Protección social

III.1. Seguros de pensiones y salud para adultos mayores

III.2. Programas asistenciales para adultos mayores

IV. A manera de conclusión: La escalera de la protección social para los adultos mayores, pros y contras

V. Bibliografía 


\section{Introducción}

El proceso de envejecimiento poblacional en México es una realidad. Estimaciones revelan que en menos de 50 años la estructura de la población mexicana se equiparará a un país envejecido, ya que la mayor parte tendrá 65 años o más. ${ }^{1}$ Esto derivado principalmente a dos grandes cambios: el aumento de esperanza de vida y la reducción de la fecundidad. De acuerdo con el Instituto Nacional de Estadística y Geografía en el último cuatrimestre de 2018 habitaban en México alrededor de 15.6 millones de personas con 60 años o más de las cuales 8.4 millones eran mujeres y 7.2 hombres. $^{2}$

Ante estos grandes cambios poblacionales, se deben tomar acciones pertinentes para hacer frente a las problemáticas que conllevan ser un país envejecido, principalmente aquellas que se encuentran íntimamente relacionadas con el mundo laboral y por consecuencia el Derecho de la Seguridad Social.

Como es bien sabido, el mercado laboral para las personas consideradas como adultas mayores tiende a reducirse y en muchos casos la posibilidad de conseguir un empleo resulta una utopía.

Por desgracia los términos vejez, adulto mayor, tercera edad, entre otros, suelen asociarse a la incapacidad de desarrollar una actividad remunerada, cerrando las puertas a millones de personas que se encuentran en esta condición limitando su derecho a la seguridad social, dejándolos al arbitrio de las políticas públicas en materia de asistencia social que los gobiernos implementen para satisfacer sus necesidades de subsistencia y atención a la salud.

En ese contexto y de una manera deductiva el presente trabajo aborda un análisis del impacto de la iniciativa internacional del piso de protección social de la Organización Internacional del Trabajo en beneficio de las personas adultas mayores, para ello se inicia con un marco teórico donde se desglosa el principal concepto de este trabajo de investigación "adulto mayor", asimismo se establecen los datos socioeconómicos que involucran a la persona de la tercera edad, esto con la finalidad de tener la noción de la problemática que vive este sector de la población en México.

Posteriormente, se analizan aspectos trascendentes del aseguramiento social que recibe este grupo etario en estado de vulnerabilidad y por supuesto la situación pensionaria a la que se enfrentan día a día los adultos mayores.

Para concluir se analiza el papel del piso de protección y su importancia en el otorgamiento de protección básica a los adultos mayores en México mediante la

\footnotetext{
${ }^{1}$ El economista, Envejecimiento de la población mexicana y su impacto en las pensiones, 2018, México, en: https://www.eleconomista.com.mx/opinion/Envejecimiento-de-la-poblacion-mexicana-y-su-impactoen-las-pensiones-20180206-0109.html, fecha de consulta: 15 de marzo de 2019.

${ }^{2}$ Instituto Nacional de Estadística y Geografía, Encuesta Nacional de Ocupación y Empleo (ENOE), población de 15 años y más de edad INEGI, 2018, México, en: https://www.inegi.org.mx/programas/enoe/15ymas/default.html\#Tabulados, fecha de consulta 1 de abril de 2019.
} 
implementación de políticas sociales. En ese apartado se desglosan los principales programas asistenciales que van enfocados a brindar prestaciones a los adultos mayores (económicas como en especie) que tienen como objetivo otorgar protección social.

Finalmente se formulan algunas conclusiones y se incluyen las fuentes de investigación consultadas.

\section{Marco teórico}

\section{II.1 Adultos mayores}

Todas las personas sin importar clase social o estilo de vida, pasan por una serie de etapas a lo largo de su vida, las cuales son clasificadas en cuatro principalmente: ${ }^{3}$ la juventud, adultez, madurez, y por último, la vejez. Por cuanto a la vejez podemos decir que se trata de un término que hace referencia a la población de personas mayores o ancianas, normalmente jubiladas y de 65 años o más. No obstante ello, podemos decir que este término ha dejado de utilizarse por los profesionales y hoy en día se utiliza el concepto personas mayores o adulto mayor. ${ }^{4}$ Es decir, es en esta etapa de la vida que como los humanos sufren modificaciones de carácter físico, dado que los huesos son más débiles, la piel se arruga, los órganos internos se han desgastado, entre otras; así como modificaciones psicológicas, afectivas, sociales como consecuencia de lo que se ha vivido a lo largo de los años.

Desde el punto de vista gramatical, viejo es un adjetivo que se refiere a la persona de edad. Comúnmente puede entenderse que es vieja la que cumplió 70 años. ${ }^{5}$ Este concepto se apoya exclusivamente en el factor edad, aun cuando, como se señalará en apartados posteriores, también podría ser una construcción social.

Según Laforest, vejez es el estado de una persona de edad avanzada, lo que implica necesariamente que su expectativa de vida está disminuida y que le queda poco tiempo de vivir. ${ }^{6}$

Por su parte el concepto envejecimiento evoca a un proceso deletéreo, progresivo, intrínseco y universal que con el tiempo ocurre en todo ser vivo a consecuencia de la interacción de la genética del individuo y su medio ambiente. Podría también definirse como todas las alteraciones que se producen en un organismo con el paso del tiempo y

\footnotetext{
${ }^{3}$ Cfr. Rocha, J., Tercera edad, ¿De qué estamos hablando?, Argentina, 2007, en: http://www.sanpablo.com.ar/rol/?seccion=articulos\&id=1475, fecha de consulta 15 de enero de 2019.

4 Hernández Luna, R., et. al., Vejez. Universidad del Golfo de México, México, 2010, en: http://es.scribd.com/doc/36045923/Que-es-la-Vejez, fecha de consulta 15 de enero de 2019.

5 Diccionario de la Lengua Española, Real Academia Española, 22a ed., en: http://buscon.rae.es/draeI/, fecha de consulta 15 de enero de 2019.

${ }^{6}$ Laforest, J., Introducción a la gerontología. El arte de envejecer. Herder, Barcelona, 1989, p. 38. 
que conducen a pérdidas funcionales y a la muerte. ${ }^{7}$ Es decir, el envejecimiento es un proceso por el que atraviesa todo ser humano por el paso del tiempo.

La Organización Panamericana de la Salud define al envejecimiento como:

...un proceso de interacción entre el individuo que envejece y una realidad contextual en proceso permanente de cambio. Así el individuo hace esfuerzos constantes por alcanzar el nivel adecuado de adaptación y equilibrio entre sus propios cambios bio-fisiológicos y aquellos otros que ocurren en el medio socioeconómico y cultural de la persona. Desde la perspectiva funcional, el anciano sano es aquel que es capaz de enfrentar el proceso de cambio a un nivel adecuado de adaptabilidad funcional y satisfacción personal. ${ }^{8}$

Es precisamente en esta etapa de la vida, en la que los adultos mayores requieren de mayor atención; no es sólo importante llegar a la tercera edad, sino bajo qué condiciones lo hacemos, no se puede olvidar que las enfermedades crónico-degenerativas como la diabetes, el cáncer o la obesidad generan altos costos a las instituciones de seguridad social, costos que no serán resarcidos, porque son erogados en personas que no contribuirán más al sostenimiento de estas instituciones puesto que por sus propias condiciones de salud no pueden laborar más y porque no existe una política de empleo eficaz dedicada a los adultos mayores, como se verá más adelante.

\section{II.1.1 Marco socio demográfico de los adultos mayores en México}

Cabe señalar que el incremento anual de personas de la tercera edad es un poco más de 292,000 personas y continuará aumentando hasta 793 mil personas en el año 2033, lo que significa que para el año 2034, por cada 100 niños habrá 166 longevos. ${ }^{9}$

Por lo que corresponde a la actividad laboral, es importante mencionar que en México a diferencia de los países desarrollados los adultos mayores siguen en actividad económica después de haber cumplido su edad de retiro, prueba de ello es que según datos del INEGI en su informe sobre "Estadísticas a propósito del día internacional de las personas de edad" (adultas mayores), durante el 2018 la población ocupada de más de 60 años representó 4.8 millones de los cuales el $49.6 \%$ trabaja por cuenta propia y el $37.6 \%$ se ocupó de manera subordinada y casi la mitad de estos (50\%) no cuenta con prestaciones de ningún tipo. ${ }^{10}$

\footnotetext{
${ }^{7}$ Rodes Texidor, J. y Guardia Massó, J., Biología del envejecimiento, Ed. Masson, Barcelona, España, 1997. pp. 3472- 3475.

${ }^{8}$ Ibídem. p. 5.

9 Benemérita Universidad Autónoma de Puebla, México necesita envejecer saludablemente, BUAP, México, 2009, en: http://www.comunicacion.buap.mx/bol_julio09/boletin-d-08julio.html, fecha de consulta 7 de enero de 2019.

${ }^{10}$ Op. cit., Instituto Nacional de Estadística y Geografía.
} 
En general, entre los adultos mayores que viven solos la proporción de personas con prestaciones son menores a la de aquellos que forman parte de un hogar familiar. ${ }^{11}$

Una parte considerable de adultos mayores subsiste de transferencias económicas proporcionadas por el Estado, de recursos que provienen de sus hogares, familiares y redes sociales. Al respecto, nuevamente el INEGI proporciona los siguientes datos: el 49.2\% de los adultos mayores que viven solos declararon recibir algún apoyo gubernamental; $28.8 \%$ recibe ayuda de familiares que residen en el país y $6.7 \%$ de personas que residen en otro país. ${ }^{12}$

En México, las instituciones de seguridad social protegen principalmente a la población que se encuentra ocupada en la economía formal y deja fuera a la población que trabaja en la economía informal, en el campo, desempleada y subempleada, y es por ello que la población de 60 años y más que tienen una pensión es muy reducida. ${ }^{13}$

De acuerdo con último censo para la Encuesta Nacional sobre uso del tiempo, los adultos mayores emplean $53.6 \%$ de las horas de la semana para atender sus necesidades y cuidados personales; la segunda actividad de este grupo poblacional es el trabajo para el mercado que es el $19.4 \%$, donde cabe señalar que de ese porcentaje las mujeres destinan en promedio $4.7 \%$ de su tiempo a dicha actividad; por su parte el trabajo doméstico representa $20.6 \%$ del tiempo de las adultas mayores, mientras que en los varones sólo $6.5 \% .^{14}$

Como pudimos observar en líneas anteriores, la longevidad es un problema que va ir incrementado año con año, lo que nos convertirá en un país en donde la mayor parte de la población será precisamente de la tercera edad, es decir, estamos siguiendo el proceso de envejecimiento europeo, quienes hoy en día su población ya es principalmente adulta y de adultos mayores.

En ese contexto, para hacer frente a la problemática que trae el envejecimiento poblacional se deben tomar acciones que permitan garantizar niveles de protección social básicas a toda la población, por ello iniciativas internacionales como por ejemplo el "piso de protección social" fungen como un medio progresivo garantizador de la seguridad social, por lo que hablar de ello resulta importante en este trabajo de investigación.

\footnotetext{
${ }^{11}$ Instituto Nacional de Estadística y Geografía, Estadísticas a propósito del día internacional de las personas de edad (adultos mayores), INEGI, México, 2018, p. 2.

12 Ídem.

${ }^{13}$ Chande Ham, Roberto, Relaciones entre envejecimiento demográfico y condiciones laborales. Ponencia para el taller de expertos en envejecimiento y políticas de empleo para grupos vulnerables. México, Secretaría de Trabajo y Previsión Social, México, en: http://envejecimiento.sociales.unam.mx/archivos/Ham_Chande.pdf?PHPSESSID=67de9ad189f0dbd5079 $854 \mathrm{c} 1 \mathrm{a} 8 \mathrm{c} 449 \mathrm{ce}$, fecha de consulta 19 de marzo de 2019.

${ }^{14}$ Instituto Nacional de Estadística y Geografía, Encuesta Nacional sobre Uso del Tiempo 2014, México, 2014, en: https://www.inegi.org.mx/programas/enut/2014/default.html\#Tabulados, fecha de consulta 29 de marzo de 2019.
} 


\section{II.2 El piso de protección social}

El piso de protección social se debe de entender como un conjunto integrado de políticas sociales diseñado para garantizar a toda persona la seguridad de los ingresos y el acceso a los servicios sociales esenciales, prestando especial atención a los grupos vulnerables, ${ }^{15}$ para ello se enfocan en cuatro garantías esenciales, que son: el acceso a la atención a la salud universal; la seguridad básica del ingreso para los niños; la seguridad del ingreso para las personas en edad activa que por sus propios ingresos no puedan satisfacer sus necesidades básicas; y la seguridad básica del ingreso a las personas adultas mayores.

El piso de protección social resulta ser uno de los mecanismos garantes de brindar la protección básica a las personas adultas mayores que se encuentren en un estado de vulnerabilidad y que por obvias razones naturales en esta etapa de la vida no pueden desempeñar un trabajo remunerado, ${ }^{16}$ desvaneciéndose la oportunidad de percibir un ingreso económico y la seguridad social ya que al estar condicionada a la laboralización, se pierde el derecho de percibir la atención médica y prestaciones económicas -pensiónque en la edad adulta resulta de suma importancia.

Ahora bien para poder establecer un piso base que garantice determinada protección social, los estados deberán adoptar políticas públicas coherentes que satisfagan los derechos de su población; en México se han implementado programas asistenciales con el fin de llegar a una protección de seguridad elemental para este grupo etario.

También se debe mencionar que estos programas resultan seriamente cuestionables debido a que son limitativos en cuanto a las prestaciones. Un claro ejemplo es la falta de certeza respecto a su continuidad, debido a que los programas asistenciales dependen directamente del presupuesto de egresos anual que recibe la federación para su aplicación, en donde año con año se pone en juicio si seguirán ofertándose cada uno de ellos.

A pesar de esto, se debe destacar que el piso de protección social es un medio dirigido a elevar la calidad de vida de los que menos tienen, así como de los más desprotegidos, donde las personas adultas mayores -por desgracia- se suman a este sector.

\section{Seguridad social vs. Protección social}

Para poder habar de este tema se deben analizar brevemente tres situaciones que se están presentando globalmente:

\footnotetext{
${ }^{15}$ Organización Internacional del Trabajo, Piso de Protección Social para una globalización equitativa e inclusiva, OIT, Suiza, 2011, p. http://www.ilo.org/wcmsp5/groups/public/@dgreports/@dcomm/@publ/documents/publication/wcms_17 6521.pdf, fecha de consulta 5 de marzo de 2019.

${ }^{16}$ En muchas ocasiones el ser una persona adulta mayor no es sinónimo de ser incapaz para realizar alguna actividad, desafortunadamente en México al llegar a esta edad, conseguir un trabajo resulta una misión imposible, pues los empleadores apuestan por mano de obra joven y barata que la mano calificada pero desgastada.
} 
1. Existen problemas latentes en la seguridad social en todo el mundo, tales como la incorporación de los grupos en estado de vulnerabilidad para aspirar a la universalidad de cobertura o los cambios poblacionales. Grupos vulnerables que son diversos en cada país, de acuerdo a su desarrollo económico y éstos pueden ser adultos mayores, niños, mujeres, jóvenes, homosexuales, trabajadores del campo, trabajadores informales, trabajadores migrantes, etc. Y cambios poblacionales como el envejecimiento poblacional, caracterizado el descenso de la tasa de natalidad que provoca la disminución del porcentaje de jóvenes y el aumento del porcentaje de adultos en edad de trabajar. Por último, el descenso conjunto de la mortalidad y de la fecundidad produce un mayor porcentaje de personas de edad, es decir, el envejecimiento de la población.

2. A los problemas globales los organismos internacionales han intentado darles soluciones globales y en ese contexto se han creado iniciativas como le Piso de Protección Social de la ONU, el cual en sí mismo constituye un gran plan de inclusión y redistribución de riqueza, pero no se debe perder de vista que acciona en cada país de forma distinta, acorde a su realidad, posibilidades y decisiones políticas, mediante diversas herramientas estatales y privadas como la asistencia social, los seguros sociales y la incorporación de la iniciativa privada y lo que estamos viendo en varios países como México es que está fortaleciendo la asistencia social y se está confundiendo con la seguridad social o con los seguros sociales. Dentro de este esquema se fortalece en México el Seguro Popular de Salud o los programas asistenciales como el de Pensión para Adultos Mayores, etc., los cuales son positivos, pero se debe dejar en claro que son programas de asistencia social, que no reúnen las características de los seguros sociales, como la exigibilidad jurídica de las prestaciones y por lo mismo no podrán ser sustitutos del aseguramiento social, sino simplemente debieran ser sólo medidas de transición.

3. La falta de exigibilidad jurídica de las prestaciones que materialicen los derechos sociales humanos como el derecho a la salud, a la educación y a la seguridad social. Desde hace una década se arrancó con el desarrollo de un eslabón más de la materialización de esas prestaciones, mediante lo que se ha llamado el Neoconstitucionalismo social, a través del cual, se fortalecen las constituciones, como norma suprema de cada nación, empoderando a los ciudadanos para lograr mediante los procesos judiciales de garantías: juicio de amparo, acciones de tutela y acciones colectivas el otorgamiento de prestaciones de seguridad social contenidas en instrumentos internacionales de derechos humanos ratificados por los países, que a su vez han servido para conformar el bloque de constitucionalidad.

$\mathrm{Y}$ en ese contexto es que se puede iniciar el debate entre seguridad social y protección social, conceptos que no son enemigos, ni contrarios, pero que no pueden ser tomados como sinónimos.

La Dra. Patricia Kurczyn señala: 
La seguridad social significa la realización efectiva de los derechos sociales a través de la aplicación de los principios de la justicia distributiva, como la realización de la justicia legal material a través del Estado de derecho social, entendido este como la fórmula para preservar la libertad a través de la dignidad humana. ${ }^{17}$

Es decir, se puede entender que la seguridad social es un componente hoy en día de la materialización de la dignidad humana; sin embargo, la tendencia de los organismos internacionales rectores en materia de seguridad social como la Organización Internacional del Trabajo, la ONU y la Asociación Internacional de la Seguridad Social han iniciado acciones en pro de la protección social, colocándola como la respuesta a los problemas que la seguridad social no ha logrado dar. Como ejemplo de ello basta citar el programa PPS o la definición de seguridad social que difunde la Asociación Internacional de Seguridad Social conlleva a la protección social y por su importancia se transcribe:

Podemos definir la seguridad social como todo programa de protección social establecido por una ley o por cualquier otro acuerdo obligatorio que ofrezca a las personas un cierto grado de seguridad de ingresos cuando afrontan las contingencias de la vejez, supervivencia, incapacidad, invalidez, desempleo o educación de los hijos. También puede ofrecer acceso a cuidados médicos curativos o preventivos. ${ }^{18}$

Es por ello que, si bien los mecanismos derivados el PPS pueden resultar en grandes beneficios para los adultos mayores al punto de solucionar la desigualdad social, constituyéndose como un mecanismo eficaz de protección social; también lo es que pueden reducirse a un grupo de programas asistencialistas que únicamente sean paliativos de los problemas actuales. En gran medida depende de las políticas de implementación que el Estado adopte para su inclusión en el país. Por lo que nos resta la duda de cuál será el camino a seguir: ¿programas asistencialistas como el seguro popular de salud o el programa prospera, pensión para adultos mayores, o verdaderos mecanismos de protección social que otorguen certeza y seguridad jurídica en el otorgamiento de sus prestaciones?

\section{III.2 Seguros de pensiones y salud para adultos mayores}

Las prestaciones que otorga la seguridad social mediante los seguros sociales en México y en especial para los adultos mayores constituyen, como se mencionó anteriormente, la satisfacción de dos de las necesidades básicas más importantes para el ser humano, y más aún cuando sus condiciones fisiológicas derivadas del envejecimiento no le permiten obtener esos satisfactores por otros medios:

\footnotetext{
${ }^{17}$ Op. cit. Kurczyn Villalobos, 2004. p. 196.

18 Asociación Internacional de la Seguridad Social, Comprender la seguridad social, en: http://www.issa.int/esl/Temas/Comprender-la-seguridad-social, fecha de consulta 5 de febrero de 2019. 
- Protección a los medios de subsistencia: mediante el otorgamiento de pensiones de diversa índole, pagos periódicos garantizados por el Estado y vitalicios, en la mayoría de los casos, y;

- Atención a la salud en los tres niveles: general, especialidad y alta especialidad, mediante las prestaciones médicas, quirúrgicas, farmacéuticas, hospitalarias y de rehabilitación de cualquier enfermedad.

Por su parte las pensiones, como todos sabemos, son el ingreso económico más necesario en la vejez y en México pueden otorgarse por invalidez, vejez o cesantía en edad avanzada, mismas que protegen a los dependientes económicos de los trabajadores en caso de que éste muera.

De acuerdo a datos emitidos por la Encuesta Nacional de Empleo y Seguridad Social en el año 2013, el 31\% de los adultos mayores se encuentra pensionado; este porcentaje es casi cincuenta por ciento mayor en los varones (60\%) que en las mujeres (40\%). En los primeros, más de la mitad de los pensionados, es decir, $55.4 \%$ son por jubilación, poco más de la tercera parte $33.6 \%$ es por retiro o vejez y sólo $5.7 \%$ es pensionado por accidente o enfermedad de trabajo. En las mujeres esta configuración cambia radicalmente, $47.9 \%$ está pensionada por viudez, $31 \%$ por jubilación y $14.1 \%$ por retiro o vejez. Tres cuartas partes $(76 \%)$ de las pensiones que se les otorgan a los adultos mayores provienen del IMSS, mientras que una sexta parte $(16.3 \%)$ las otorga el ISSSTE y $7.7 \%$ otras instituciones. ${ }^{19}$

\section{III.3 Programas asistenciales para adultos mayores}

El estado mexicano ha implementado determinados programas asistenciales que van enfocados a garantizar cierto grado de protección social a los adultos mayores, ya sea garantizando acceso a prestaciones económicas o en especie, dentro de estos encontramos los siguientes vigentes en el 2019:

a) Pensiones para el bienestar de las personas adultas mayores: Este programa a cargo de la Secretaría de Bienestar garantiza principalmente prestaciones de naturaleza económica a este sector de la población, teniendo como objetivo contribuir al bienestar social e igualdad de la población adulta mayor, a través del otorgamiento mensual de una pensión no contributiva. ${ }^{20}$ a personas mayores de 65 años que residan en territorios considerados como indígenas y personas mayores de 68 años que residan en la república mexicana.

\footnotetext{
${ }^{19}$ Gobierno de la República, ¿Quiénes y cuántos mexicanos tienen acceso a una pensión, en: https://www.gob.mx/cms/uploads/attachment/file/272221/19_Blog_ENIGH_pensiones_VF.pdf, fecha de consulta 15 de marzo de 2019, No se cuenta con información oficila más actualizada.

${ }^{20}$ Diario Oficial de la Federación, ACUERDO por el que se emiten las Reglas de Operación de la Pensión para el Bienestar de las Personas Adultas Mayores, para el ejercicio fiscal 2019. en: https://dof.gob.mx/nota_detalle.php?codigo=5551445\&fecha=28/02/2019, fecha de consulta 1 de abril de 2019.
} 
La suma que se les otorga a los beneficiarios del programa para el año 2019 es de $\$ 1,275.00$ pesos mensuales ${ }^{21}$ (\$67.21 USD) pagaderas de manera bimestral, y en dado caso del fallecimiento del beneficiario se otorgara un pago único por la misma cantidad a sus beneficiarios por concepto de pago de marcha.

A través de este programa se pretende establecer una mejor calidad de vida de las personas adultas mayores que carecen de alguna entrada económica o en su defecto si la fuente económica que perciben es inferior al monto establecido en los criterios del programa poder ser beneficiarios para obtener una suma extra que les permita satisfacer sus necesidades básicas, este programa se encuentra estrechamente vinculado a una de las cuatro garantías del piso de protección social, el aseguramiento del ingreso de las personas adultas mayores.

Asimismo, presta servicios vinculados a la atención de la salud y de recreación para brindarles determinada protección social, esto mediante las siguientes acciones:

I. Promoción de los Derechos Humanos, en específico derechos de las y los Adultos Mayores

II. Desarrollo Personal

III. Cuidados de la Salud

IV. La obtención de la Credencial del INAPAM.

V. El acceso a los servicios de salud (Seguro Popular).

VI. La rehabilitación, acondicionamiento y equipamiento de Casas de Día para la atención de Adultos Mayores. ${ }^{22}$

Cifras proporcionadas a través del sexto informe presidencial de gobierno (2018) establecen que hasta junio del 2018 más de 4.9 millones de personas se encontraban afiliadas al programa. ${ }^{23}$

b) Prospera: Este es el programa asistencial por excelencia en México que ha servido como ejemplo para la implementación del piso de protección social a los demás países latinoamericanos. Destacan las transferencias económicas condicionadas dirigidas

\footnotetext{
${ }^{21}$ El tipo de cambio utilizado para este informe es de \$ 18.97 pesos mexicanos $=1$ USD, datos del Banco de México el 8 de marzo de 2019, disponible en: Banco de México, Tipo de cambio, México, Banco de México, 2019, en: http://www.anterior.banxico.org.mx/portal-mercado-cambiario/, fecha de consulta 8 de marzo de 2019.

${ }^{22}$ Op. cit. Diario Oficial de la Federación.

23 Presidencia de la Republica, Sexto informe de gobierno, México, 2018, p. 145. en: http://cdn.presidencia.gob.mx/sextoinforme/informe/6_IG_Anexo_Estadistico.pdf, fecha de consulta 5 de marzo de 2019. 
a las familias mexicanas que se encuentran en condiciones de desventaja y desigualdad apoyándolos en el fortalecimiento de su salud, alimentación y educación. ${ }^{24}$

Es uno de los programas de asistencia social que, a pesar del cambio de sexenios en el ejecutivo federal y transiciones de poder sigue estando en operación y es uno de los más completos tanto en el ramo prestacional como el público al cual se encuentra dirigido.

Prospera resalta por ser un programa integral, debido a que se garantizan las prestaciones básicas a la población beneficiaria para la construcción de un piso base de protección social, teniendo como objetivo dar prioridad al desarrollo social mediante el apoyo a la alimentación, salud y educación. ${ }^{25}$

Dentro del ramo prestacional que otorga este programa se encuentran divididos por 4 componentes:

I. El alimentario, donde se otorga apoyo económico para la alimentación de la familia;

II. Educación, consistente en apoyo con becas para los jóvenes que se encuentren estudiando en la educación básica, así como apoyo para la compra de útiles escolares;

III. Salud, encaminada a la atención médica y en algunos casos hospitalarios;

IV. Vinculación, tiene como finalidad incluir al campo laboral formal a los jóvenes que son beneficiarios del programa posterior a la terminación de sus estudios de preparatoria, así como el otorgamiento de créditos baratos, seguro de vida, educación financiera entre otros a las jefas de familia.

De lo anterior se puede afirmar que para el sector poblacional considerado adulto mayor se otorgan básicamente dos prestaciones: la atención a la salud y la entrega de recursos económicos para su subsistencia, haciendo precisión que se deben de cumplir con ciertos requisitos para ser un beneficiario.

Una crítica al programa es que el estado mexicano no ha implementado plenamente el piso de protección social o en su defecto ha optado por hacer caso omiso por conveniencia, ya que los programas sociales tienden a ser utilizados para condicionar la entrega de las transferencias económicas a cambio de votos. Esto sumado al cambio presidencial en el año 2018, y la consiguiente reducción en su financiamiento apunta a que el programa dejará de existir o al menos si reducirá considerablemente su importancia en este sexenio.

\footnotetext{
${ }^{24}$ Secretaría de Bienestar, En PROSPERA celebramos nuestro $4^{\circ}$ Aniversario., 2019, México, en: https://www.gob.mx/prospera/articulos/en-prospera-celebramos-nuestro-4-aniversario?idiom=es, fecha de consulta 30 de marzo de 2019.

${ }^{25}$ Cfr. Op. cit., Diario Oficial de la Federación. 
c) El seguro popular de salud: El objetivo de este programa es proteger a toda la población que no cuente ya con un seguro social de gastos médicos, buscando de este modo que todos los integrantes de las familias afiliadas al seguro popular tengan acceso a los servicios de salud, médicos, hospitalarios, farmacéuticos y quirúrgicos. ${ }^{26} \mathrm{~A}$ través de este programa el estado mexicano busca dar cobertura universal en materia de atención a la salud a toda la población mexicana que no se encuentre asegurada bajo un instituto de seguridad social, siendo beneficiado con las prestaciones de médicas, hospitalarios, farmacéuticos y quirúrgicos. ${ }^{27}$

Para poder ser beneficiario de este programa se tiene que cubrir con ciertos requisitos mínimos como: Residir en las regiones de cobertura del Seguro Popular de Salud, no ser derechohabiente de la seguridad social, optar voluntariamente por afiliarse, cubrir la cuota de afiliación correspondiente. ${ }^{28}$

El mecanismo de financiamiento de este programa es cubierto mediante subsidio federal proveniente de los recursos fiscales del presupuesto de egresos de la federación, al igual de las cuotas de los beneficiarios del programa por concepto de afiliación y las de recuperación por concepto de servicios médicos.

Es de resaltar que el seguro popular no es una institución de seguridad social pues las prestaciones que brinda este programa son consideradas no contributivas y por consecuencia las limitaciones de los beneficios que se pueden esperar de esta frente a una institución de seguridad social son enormes e incomparables, asimismo los beneficios o prestaciones no pueden ser exigibles mediante algún recurso administrativo sencillo y eficaz, pues se debe recordar que al ser un programa asistencial carece de exigibilidad jurídica lo que genera una incertidumbre para los beneficiarios en caso de alguna violación de sus derechos.

Asimismo se debe estar consciente que para ser atendido dentro de uno de los centros médicos que establece el programa, el padecimiento a tratar deberá encontrarse dentro del Catálogo Universal de Servicios de Salud, ${ }^{29}$ en este catálogo se establecen todos los padecimientos, tratamientos, intervenciones quirúrgicas, medicamentos, etcétera, que la red del seguro popular cubre a las personas beneficiarias del programa, por lo tanto si el padecimiento, intervención o medicamento no se encuentra estipulado en el multicitado catálogo, la institución no esa obligada prestar el servicio o garantizarte el medicamento.

En ese contexto, el seguro popular (como en muchos de los programas asistenciales) carece de seguridad y certeza por parte de los beneficiarios para recibir la atención

\footnotetext{
${ }^{26}$ Seguro popular, El seguro popular, México, 2015, en: http://www.seguropopular.org/, fecha de consulta 15 de marzo de 2019.

27 Ídem.

${ }^{28}$ Secretaría de Salud, Reglas operacionales, México, 2018, en: http://www.salud.gob.mx/unidades/dgpfs/reglas_operacion/4_lineamientos_generales.htm, fecha de consulta: de 12 de marzo de 2019.

${ }_{29}$ Comisión Nacional de Protección Social en Salud, Universal de Servicios de Salud, CAUSES 2019, México, 2019, en: https://www.gob.mx/salud\%7Cseguropopular/documentos/catalogo-universal-deservicios-de-salud-causes-2018-153111, fecha de consulta 9 de marzo de 2019.
} 
médica, dejándolos en la incertidumbre si en algún momento llegaran a padecer una enfermedad que se encuentre fuera del catálogo.

d) Programa de apoyo al empleo: Es un programa a cargo de la Secretaría del Trabajo y Previsión Social relativamente nuevo, que entró en vigor el primero de enero de 2017.

A través de este programa se busca facilitar la vinculación laboral a las personas debido a las dificultades que enfrentan la oferta y demanda de empleo para entrar en el mercado laboral, pues existen diversos factores que impiden la incorporación a la vida económica de los diferentes sectores de la población como la falta de promoción de las vacantes disponibles para su ocupación; la insuficiencia de conocimientos, habilidades y destrezas laborales de los Buscadores de empleo; ${ }^{30}$ así como pertenecer al grupo poblacional de adultos mayores y personas con discapacidad.

Conscientes de que ser una persona adulta mayor no significa estar impedido para realizar una actividad remunerada, pero si se presenta la dificultad para poder encontrar una oportunidad laboral, el programa de apoyo al empleo tiene como finalidad brindar atención a la población buscadora de empleo, mediante la prestación de Servicios de Vinculación Laboral, apoyos económicos o en especie para capacitación, autoempleo, movilidad laboral y apoyo a repatriados, con la finalidad de facilitar su colocación en un puesto de trabajo. ${ }^{31}$

$A$ través de este programa se busca la reinserción a la económica formal a la población mexicana, donde se encuentra incluido uno de los grupos sociales más vulnerables: las personas adultas mayores.

Los mecanismos implementados para lograr la vinculación a las personas son a través de observatorios laborales, talleres para buscadores de empleo, los sistemas estatales de empleo, centros de intermediación laboral, periódico de ofertas de empleo, entre otros.

De esta forma se brinda atención enfocada a las necesidades específicas de la población beneficiaria, la cual consiste en el otorgamiento de servicios de vinculación laboral, así como apoyos económicos para: fortalecer habilidades laborales; promover la ocupación por cuenta propia; facilitar la movilidad laboral, y procurar la colocación de repatriados. ${ }^{32}$

\section{A manera de conclusión: La escalera de la protección social para los adultos mayores, pros y contras.}

Considerando los puntos anteriores podemos afirmar que una de las ventajas más importantes el piso de protección social es fungir como un medio progresivo garantizador

\footnotetext{
${ }^{30}$ Diario Oficial de la Federación, ACUERDO mediante el cual se establecen las Reglas de Operación del Programa de Apoyo al Empleo, SEGOB, México, 2019, en: https://www.gob.mx/cms/uploads/attachment/file/441291/ROPAE_2019_DOF.PDF, fecha de consulta 4 de abril de 2019.

31 Ídem.

32 Ídem. 
de la seguridad social en la población mexicana, particularmente para aquellos grupos vulnerables carentes de cualquier tipo de protección social, dentro de los cuales sin duda encontramos a las personas adultas mayores.

Sin embargo; se debe estar plenamente consciente de que los programas de asistencia social pueden ser un medio eficaz para garantizar en primer plano una protección social básica, para que con posterioridad, se fortalezca a la población vulnerable y pueda escalar por si misma a niveles más altos de protección, como al aseguramiento social laboral.

Atendiendo que, en México, existen tres pisos de protección social, el primero; garantizado por las prestaciones derivadas de la asistencia social, segundo; los beneficios que derivan de la seguridad social a través de los seguros sociales y; tercero, la contratación de seguros privados (seguro médico), que en su conjunto forman la escalera de pisos de protección social.

Para que estos programas asistenciales tengan el impacto esperado, es necesario modificar las características jurídicas que tienen actualmente. Por ejemplo, necesitan un blindaje anticorrupción, y además que sean exigibles a través de mecanismos jurídicos establecidos en una ley.

Pese a ello, estos programas asistenciales resultan ser medios directos de protección para los adultos mayores que por cuestiones de la edad misma, no puede allegarse de los medios indispensables para su subsistencia y una existencia digna.

\section{Fuentes de investigación}

Asociación Internacional de la Seguridad Social, Comprender la seguridad social, en: http://www.issa.int/esl/Temas/Comprender-la-seguridad-social

Benemérita Universidad Autónoma de Puebla, México necesita envejecer saludablemente, BUAP, México, en: http://www.comunicacion.buap.mx/bol_julio09/boletin-d-08julio.html

Chande Ham, Roberto, Relaciones entre envejecimiento demográfico y condiciones laborales. Ponencia para el taller de expertos en envejecimiento y políticas de empleo para grupos vulnerables. México, Secretaría de Trabajo y Previsión Social, México, en:http://envejecimiento.sociales.unam.mx/archivos/Ham_Chande.pdf?PHPSESSID=67 de9ad189f0dbd5079854c1a8c449ce

Comisión Nacional de Protección Social en Salud, Universal de Servicios de Salud, CAUSES 2019, México, 2019, en: https://www.gob.mx/salud\%7Cseguropopular/documentos/catalogo-universal-deservicios-de-salud-causes-2018-153111 
Diario Oficial de la Federación, ACUERDO mediante el cual se establecen las Reglas de Operación del Programa de Apoyo al Empleo, SEGOB, México, 2019, en: https://www.gob.mx/cms/uploads/attachment/file/441291/ROPAE_2019_DOF.PDF

ACUERDO por el que se emiten las Reglas de Operación de la Pensión para el Bienestar de las Personas Adultas Mayores, para el ejercicio fiscal 2019. en: https://dof.gob.mx/nota_detalle.php?codigo=5551445\&fecha=28/02/2019

Diccionario de la Lengua Española, Real Academia Española, 22a ed., en: http://buscon.rae.es/draeI/

El economista, Envejecimiento de la población mexicana y su impacto en las pensiones, 2018, México, en: https://www.eleconomista.com.mx/opinion/Envejecimiento-de-lapoblacion-mexicana-y-su-impacto-en-las-pensiones-20180206-0109.html

Gutiérrez Carmona, A., Autoconcepto y revisión de vida en la vejez. México, p. 4. en: http://catarina.udlap.mx/u_dl_a/tales/documentos/lps/gutierrez_c_a/capitulo1.pdf

Hernández Luna, R., et. al., Vejez. Universidad del Golfo de México, México, 2010, en: http://es.scribd.com/doc/36045923/Que-es-la-Vejez

https://www.gob.mx/cms/uploads/attachment/file/272221/19_Blog_ENIGH_pensiones_ $V F . p d f$

Instituto Nacional de Estadística y Geografía, Encuesta Nacional de Ocupación y Empleo (ENOE), población de 15 años y más de edad INEGI, 2018, México, en: https://www.inegi.org.mx/programas/enoe/15ymas/default.html\#Tabulados

, Encuesta Nacional sobre Uso del Tiempo 2014, México, 2014, en: https://www.inegi.org.mx/programas/enut/2014/default.html\#Tabulados

Estadísticas a propósito del día internacional de las personas de edad (adultos mayores), INEGI, México, 2018, p. 2.

Laforest, J., Introducción a la gerontología. El arte de envejecer. Herder, Barcelona, 1989 , p. 38.

Organización Internacional del Trabajo, Piso de Protección Social para una globalización equitativa e inclusiva, OIT, Suiza, 2011, p. 9, en: http://www.ilo.org/wcmsp5/groups/public/@dgreports/@dcomm/@publ/documents/pub lication/wcms_176521.pdf

Presidencia de la Republica, Sexto informe de gobierno, México, 2018, p. 145. en: http://cdn.presidencia.gob.mx/sextoinforme/informe/6_IG_Anexo_Estadistico.pdf 
Rocha, J., Tercera edad, ¿De qué estamos hablando?, Argentina, 2007, en: http://www.san-pablo.com.ar/rol/?seccion=articulos\&id=1475

Rodes Texidor, J. y Guardia Massó, J., Biología del envejecimiento, Ed. Masson, Barcelona, España, 1997. pp. 3472- 3475.

Secretaría de Bienestar, En PROSPERA celebramos nuestro $4^{\circ}$ Aniversario., 2019, México, en: https://www.gob.mx/prospera/articulos/en-prospera-celebramos-nuestro-4aniversario?idiom $=\mathrm{es}$

Secretaría de Salud, Reglas operacionales, México, 2018, en: http://www.salud.gob.mx/unidades/dgpfs/reglas_operacion/4_lineamientos_generales.ht $\mathrm{m}$

Seguro popular, El seguro popular, México, 2015, en: http://www.seguropopular.org/ 